\title{
Developing an Integrated Model Centred on Artificial Intelligence to Effectively Predict and Analyse Climate Change Including Global Warming ${ }^{1}$
}

\author{
Sehaj Bedi \\ Amity University, Noida
}

DOI: 10.37648/ijrst.v10i04.006

Received: 07th August, 2020; Accepted: 10 ${ }^{\text {th }}$ October, 2020; Published: $30^{\text {th }}$ October, 2020

\begin{abstract}
An increase in average temperature worldwide is called global warming. Regular occasions and human exercises are accepted to be adding to expanding normal worldwide temperatures. Long haul impacts of environmental change are continuous out of control fires, longer times of the dry season in certain areas and an increment in the number, term and power of typhoons. Global warming forecasting can be vital in the rural, energy and clinical space. This paper assesses the exhibition of a few calculations in yearly a worldwide temperature alteration anticipation from recently estimated values over the Globe. The primary test is making a solid, effective and exact information model on an enormous dataset and noticing the connection between the normal yearly temperatures and potential variables adding to Global Warming, for example, the convergence of Greenhouse gases. The information is anticipated and determined utilizing straight relapse for acquiring the most important accuracy for ozone-depleting substances and temperature contrasted with different strategies. After noticing the analysed and expected information, global warming can be reduced relatively inside a couple of years. The decrease of worldwide temperature can assist us with forestalling unsafe long-haul impacts of global warming and Climate change.
\end{abstract}

\section{INTRODUCTION}

The greenhouse effect is caused due to the average increase in temperature. Certain gases in the air behave like glass in a nursery, permitting daylight to warm the Earth's surface yet catching the hotness as it emanates once more into space. As the ozonedepleting substances develop in the climate, the Earth gets more burning. This cycle prompts a quick change in the environment, otherwise called the environment. This issue is encouraging fast climatic switches generally up the world. The Earth's normal temperature is $1{ }^{\circ} \mathrm{C}$ higher than 100 years prior.
Numerous researchers expect that in the following 200 years, the normal temperature will increment by roughly $6{ }^{\circ} \mathrm{C}$ than the normal temperature. It has been developing starting around 1990 caused because of the outflow of ozone-depleting substances from presentday industry, vehicle discharge, copying of petroleum derivatives. And so forth. If the temperature increments, many Forests would be diminished, nursery gasses would increment, and Sea levels would likewise ascend to a gigantic degree, consequently lowering numerous urban areas.

\footnotetext{
${ }^{1}$ How to cite the article: Bedi S., Developing an Integrated Model Centred on Artificial Intelligence to Effectively Predict and Analyse Climate Change Including Global Warming, IJRST, Oct-Dec 2020, Vol 10, Issue 4, 43-48, DOI: http://doi.org/10.37648/ijrst.v10i04.006
} 


\section{PROPOSED IDEA}

The principal objective of this examination is to dissect and anticipate the ascent of temperature consistently in different landmasses for appx. 100-150 years dependent on information recorded in Kaggle.

The particular target of this work is to foresee the temperature and ozone harming substances focus for the following decade and envision the forecast for simplicity of comprehension.

\section{PREVIOUS WORK}

A couple of kinds of examinations are being done regarding climate forecast, precipitation expectation, and temperature expectation. A portion of those thoughts is taken for reference purposes.

AI Applied to Weather Forecasting" [1], "Confined Precipitation Forecasts from a Numeric Weather Prediction Model Using Artificial Neural Networks" [2], "Neural Network Local Forecasting with Weather Ensemble Predictions" [3]. "A half and half Double Feedforward Neural Network for Suspended Sediment Load Estimation" [4] is an expectation-based paper. However, they don't zero in on temperature or a dangerous atmospheric deviation. In this task, I primarily anticipate the effect of a difficult atmospheric variation for the past couple of years.

\section{PROPOSED APPROACH OF GLOBAL WARMING SYSTEM}

There are numerous calculations to foresee information like Support Vector Regression, Linear Regression, Multiple Regression, etc. We have attempted different calculations to get the most noteworthy exactness. Every one of the calculations has various methods of working the analysis as examined below: -

\section{A. Multiple Regression}

It is a strategy that connects a reliant variable and at least one autonomous factor. The reliant variable is displayed as an element of the distinctive independent factors. Here the essential distinction from the linear Regression is the free factor could conceivably be multiple, staying any remaining things are something similar. Here, the straight indicator work is additionally used to make an object of that capacity and utilized for other expectations.

\section{B. Linear Regression}

It is a technique that gives a connection between a reliant variable or scalar variable and an independent variable or logical variable. In this technique, the associations are demonstrated utilizing a direct indicator work. Here the information is prepared by this technique. In the wake of making the thing, the data is a guage for what's to come. The direct indicator work creates an object of that capacity and expectation.

\section{Support Vector Regression}

It is a part of the Support Vector Machine. SVR follows a similar rule, which is trailed by SVM. The expectation strategy is hard to help vector relapse distinguished with different techniques. The algorithm is more confused. Among every one of the innovations, more precision is seen in Linear Regression. For that, in this paper, Linear Regression is utilized. The complexity of Linear Regression is likewise relatively a lot lesser than different innovations. A few modules are needed to foster the Global Warming Prediction System. Those modules are momentarily clarified underneath:

Can't foresee this crude information straightforwardly.

a. Data Collection:

In this module, the raw information is gathered from the various informational indexes. Then, at that point, the informative index is changed according to need. In this way, it is expected to pre-process.

b. Information Pre-handling :

In this module, the information is cleaned. After cleaning the report, the data is assembled according to the requirement. This group of information is known as information clustering. Then, at that point, check to assume there is any missing value in the informational index. Assuming there is some missing value, change it by any default esteem. From that point forward, it is completed, taking any information needed to change its arrangement. That entire cycle before the expectation is known is pre-processing data. The data is utilized for the anticipation and counting step from that point forward.

c. Information Prediction and anticipating:

Can make this forecast in any cycle that is referenced previously. Yet, the Linear Regression calculation scores more expectation accuracy than the other 
analysis. Thus, the direct relapse strategy is utilized to anticipate this venture. In this progression, the prehandled information is taken for the expectation. The pre-processing information is divided for train and test purposes. Then, at that point, a proactive item is made to anticipate the test esteem, which is prepared by the prepared value. Then, at that point, the thing is utilized to figure out information for not many years.

d. Perception:
In this progression, the anticipated and predicted information is utilized to give a graphical interface independently. From the beginning, the normal data is plotted in a diagram independently with the assistance of the matplot library. Then, at that point, the anticipated temperature information is plotted in a graph with a legitimate scale. Then, at that point, the ozone-damaging substances anticipated data are plotted in a solitary diagram with a fitting scale.

\section{PROPOSED APPROACH FLOW DIAGRAM}

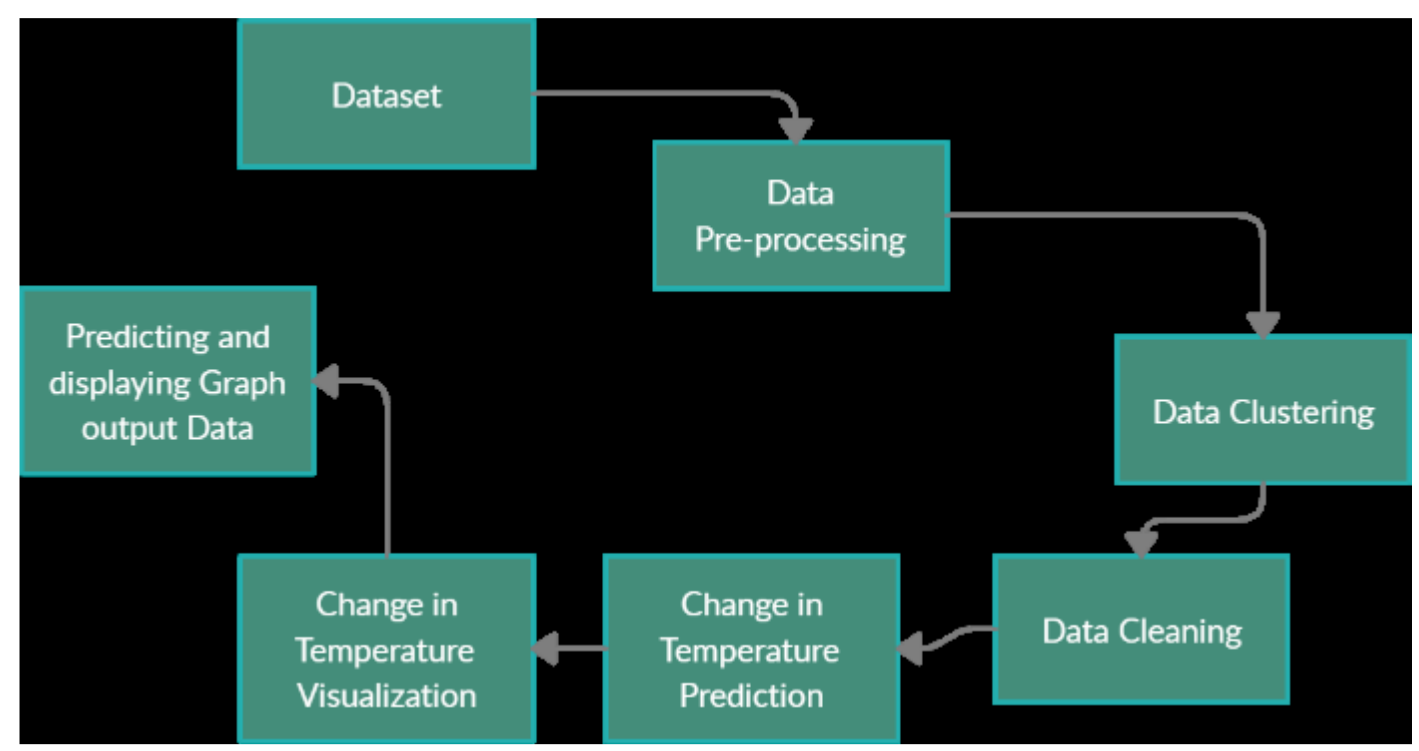

Fig 1: Flow Diagram

\section{VISUAL OUTPUT}

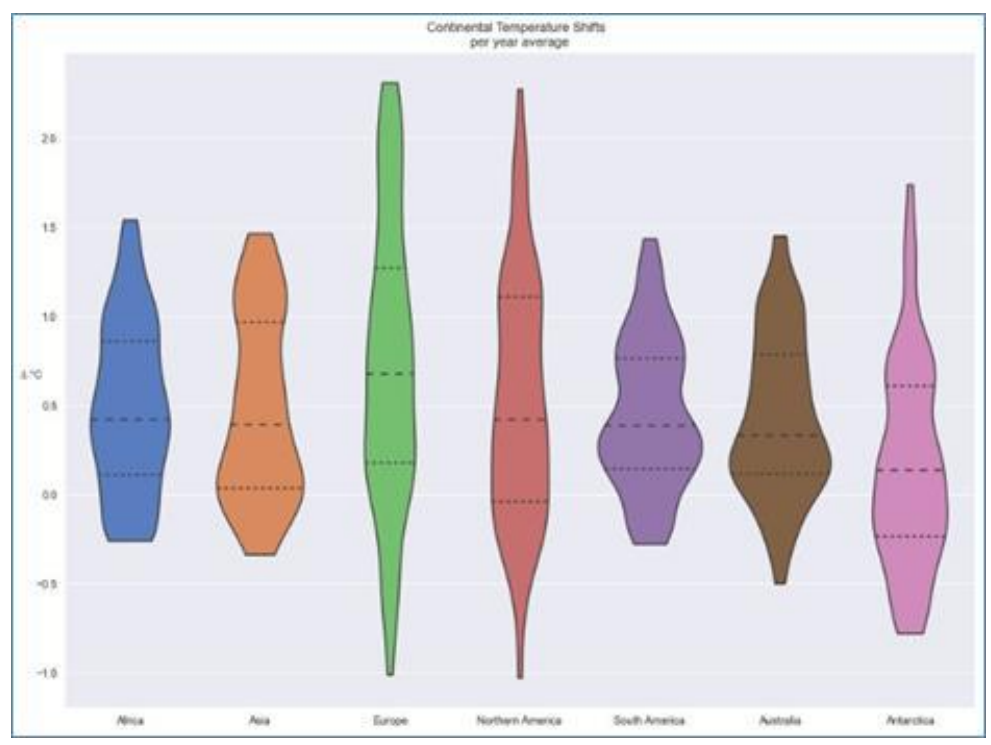

Figure 2. Continent temperature shifts average (per year) 


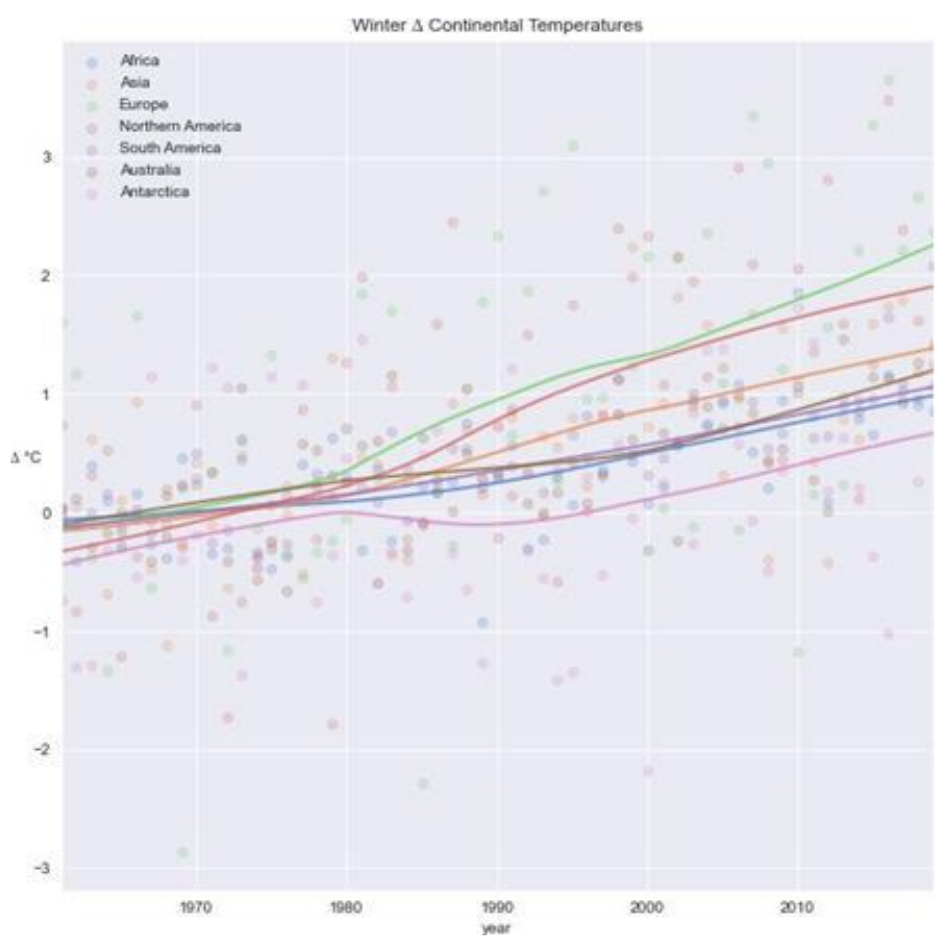

Figure 3. Change in Winter Continental Temperature

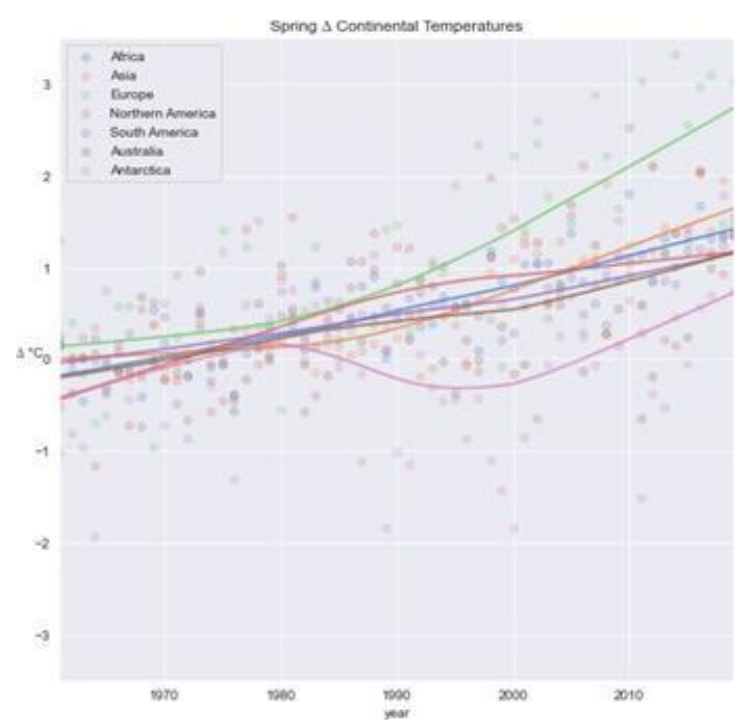

Figure 4. Change in Spring Continental Temperature 


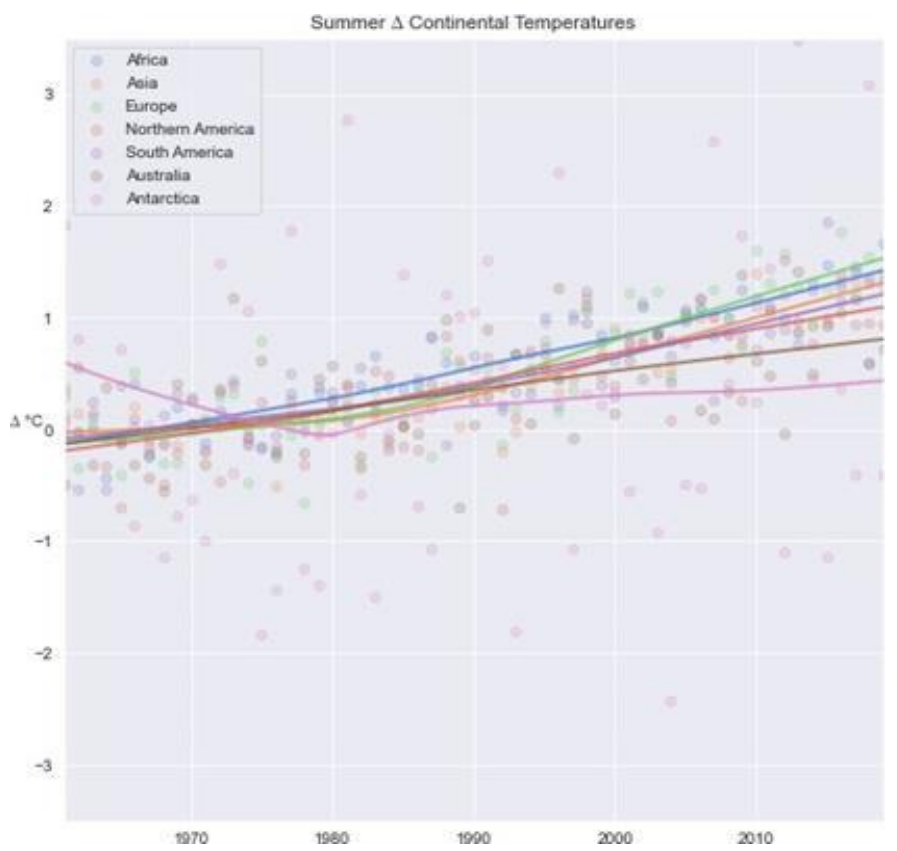

Figure 5. Change in Summer Continental Temperature

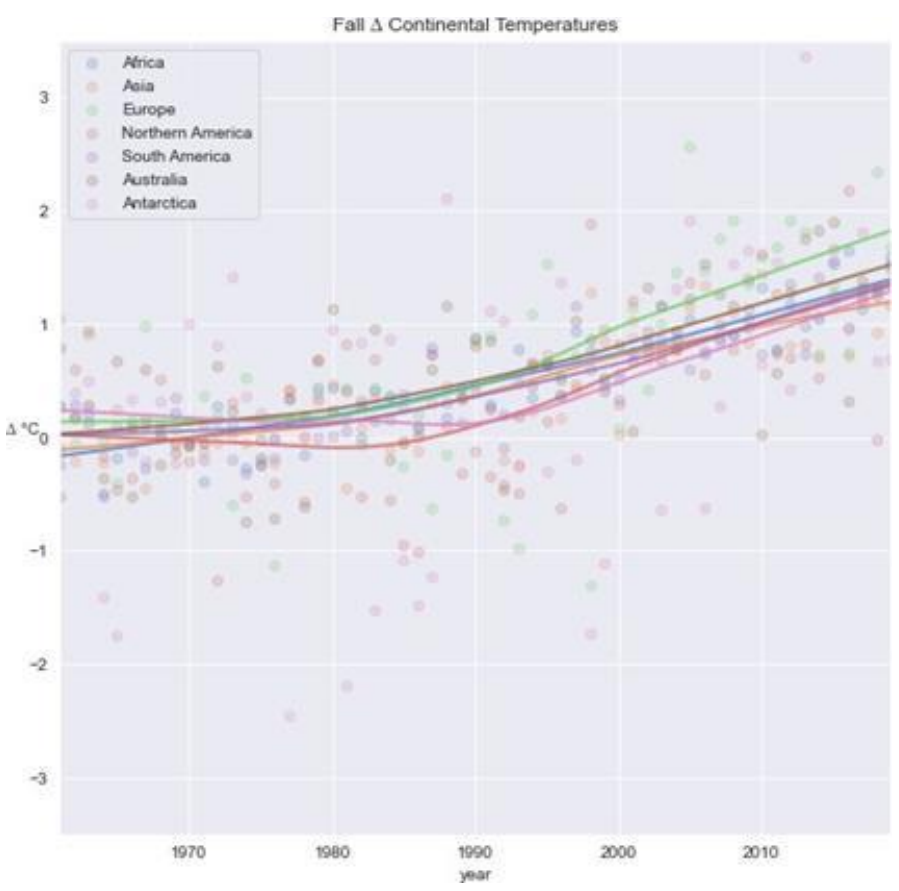

Figure 6. Change in Fall Continental Temperature 


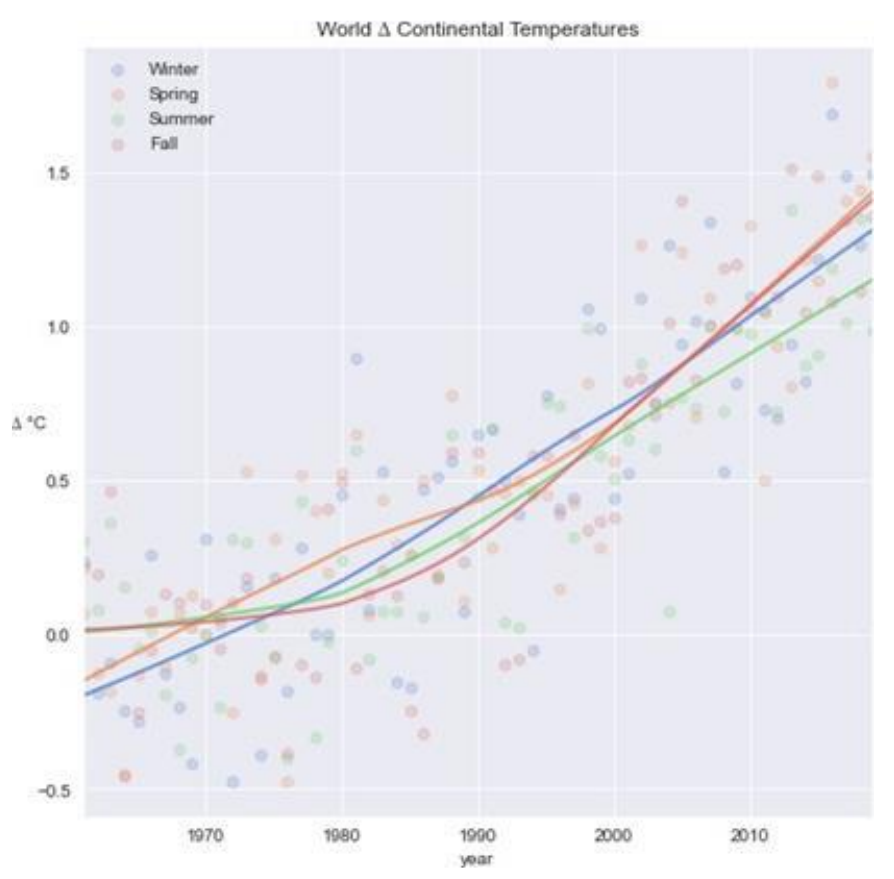

Figure 7. Change in World Continental Temperature

\section{RESULT}

After fruitful investigation of the temperature, higher accuracy is obtained for the temperature forecast and ozone-depleting substances expectation. After the productive research, we can imagine the adjustment of yearly worldwide temperature and what it can mean for us. After seeing the diagram for the prediction, we can say that the temperature and actual variables answerable for an unnatural weather change will build step by step in a straight example. The aftereffect of this, an Earth-wide temperature boost will turn out to be increasingly deadly.

\section{CONCLUSION}

In this paper, the information of 100-150 years is examined. Python is utilized to explore and picture the temperature and ozone-depleting substances for the following 10 years on average. The MatPlot library is used to plot the anticipated and the forecasted information. On seeing the accompanying statement, the temperature continues to increase worldwide, and the charts show a similar change. Accordingly, assuming the temperature continues expanding at a similar rate, it can cause a tremendous effect from one side of the planet to the other.

\section{REFERENCES}

[1]. "Machine Learning Applied to Weather Forecasting” by Mark Holmstrom, Dylan Liu, Christopher Vo; 2016

[2]. "Localized Precipitation Forecasts from a Numeric Weather Prediction Model Using Artificial Neural Networks" by R. J. Kuligowski and A.

[3]. P. Barros; American Meteorological Society (Vol - 13)

[4]. I. "Neural Network Local Forecasting with Weather Ensemble Predictions" by J. W. Taylor and R. Buizza; IEEE Transactions and Power Systems (Vol - 17); August-2002

[5]. “A hybrid Double Feedforward Neural Network for Suspended Sediment Load Estimation” by Xiao Yun Chen and Kwok Wing Chau; Water Resources Management (Vol - 30); May-2016 